\title{
Negative impact of neoadjuvant hormonal therapy on detecting biochemical recurrence after radical prostatectomy
}

\author{
Kazuhiro Matsumoto ${ }^{1}$ (D) Naoya Niwa ${ }^{1} \cdot$ Takeo Kosaka $^{1} \cdot$ Toshikazu Takeda $^{1} \cdot$ Yota Yasumizu $^{1} \cdot$ Nobuyuki Tanaka $^{1}$. \\ Shinya Morita ${ }^{1} \cdot$ Ryuichi Mizuno $^{1} \cdot$ Toshiaki Shinojima $^{1} \cdot$ Hiroshi Asanuma $^{1} \cdot$ Mototsugu Oya $^{1}$
}

Received: 18 January 2021 / Accepted: 21 May 2021 / Published online: 4 June 2021

(C) Japan Society of Clinical Oncology 2021

\begin{abstract}
Background Routine use of neoadjuvant hormonal therapy (NHT) before radical prostatectomy (RP) is not recommended, but it is sometimes performed to reduce the prostate size and tumor volume or to prevent tumor progression during the wait times for surgery in clinical practice. On the other hand, the impact of NHT on the pattern of biochemical recurrence (BCR) is unknown.

Methods We retrospectively examined 1749 consecutive patients who underwent RP between 1996 and 2017. Among the patients who met the inclusion criteria, BCR developed in 240 of non-NHT patients and in 120 of NHT patients during the mean follow-up period of 6.9 years. We examined the impact of NHT on the PSA-doubling time (DT) following BCR at different times after RP.

Results The median PSA-DTs in non-NHT patients who experienced BCR in the first year after surgery, between 1 and 2 years, between 2 and 3 years, between 3 and 4 years, between 4 and 5 years, and at $>5$ years were 5.5, 8.8, 11.3, 17.7, 18.2, and 18.4 months, respectively. On the other hand, those in NHT patients were 1.4, 4.1, 9.1, 13.4, 27.2, and 19.3 months, respectively. The differences of PSA-DTs in the first year after surgery $(p<0.001)$ and between 1 and 2 years $(p=0.005)$ were significant between non-NHT and NHT patients.

Conclusion Patients who received NHT had a higher risk of a rapid PSA increase when they experienced BCR, especially within 2 years after RP. In order to not miss the optimal timing of salvage treatment for BCR, intensive PSA follow-up is necessary.
\end{abstract}

Keywords Radical prostatectomy · Biochemical recurrence $\cdot$ Neoadjuvant androgen deprivation therapy $\cdot$ PSA $\cdot$ PSAdoubling time

\section{Introduction}

Since the introduction of serum prostate-specific antigen (PSA) testing, the incidence of clinically localized prostate cancer has markedly increased and radical prostatectomy (RP) is now a mainstream of curative therapy [1]. RP is considered oncologically successful when the serum level of PSA decreases to $<0.2 \mathrm{ng} / \mathrm{mL}$ after surgery. Biochemical recurrence (BCR) after RP is commonly diagnosed by detecting an increase in the PSA level without clinical or

Kazuhiro Matsumoto

kazz_matsumoto@yahoo.co.jp

1 Department of Urology, Keio University School of Medicine, Shinanomachi 35, Shinjuku-ku, Tokyo 160-8582, Japan radiographic evidence of disease, and generally defined as two successive PSA levels of $>0.2 \mathrm{ng} / \mathrm{mL}$ [2]. Not all patients who have BCR after RP have a poor prognosis [3], but conversely, all patients who develop distant metastasis and die due to prostate cancer later inevitably develop BCR in advance. Therefore, PSA monitoring is essential for surveillance after RP. As early salvage therapy for BCR is advantageous [4-7], it is important to detect the increase in PSA without delay. Patients should be followed up closely during the early phase after RP when aggressive disease recurrence can develop. The risk of rapid BCR gradually decreases over time and the follow-up frequency can be reduced in the late phase [8-10].

Routine use of neoadjuvant hormonal therapy (NHT) before RP is not recommended due to its cost, side effects [11], and no clear evidence of improved prognosis [12-14]. 
However, it is sometimes performed to reduce the prostate size and tumor volume or to prevent tumor progression during the wait times for surgery in clinical practice. Furthermore, the worldwide COVID-19 pandemic from 2020 caused RP to be postponed as a non-priority surgery, and overuse of NHT to stall for time until surgery is performed [15]. However, NHT may mask the PSA increase by residual prostate cancer after RP because it can cause the PSA to decrease for a certain period. Therefore, we must be careful when setting the interval of PSA follow-up after RP for patients with NHT in order not to miss the rapid increase in PSA.

For NHT, a luteinizing hormone releasing hormone (LHRH) agonist/antagonist and/or antiandrogen drug are usually selected. Several previous studies demonstrated that testosterone recovery to the normal level after hormonal therapy took several months [16-23]. On the other hand, the duration that NHT affects the pattern of BCR after RP has not been examined.

The PSA-doubling time (PSA-DT) is defined as the number of months it takes for the PSA level to double from the baseline value [24]. The increase in PSA after surgery is thought to reflect growth of the residual tumor and the PSADT remains relatively constant over time after BCR [25]. In the present study, we focused on the PSA-DT following BCR at different times after RP. We compared PSA-DTs between patients with and without NHT to elucidate the impact of NHT on the pattern of BCR.

\section{Materials and methods}

After institutional review board approval, we retrospectively examined 1749 consecutive patients who underwent RP between 1996 and 2017. We excluded patients who received adjuvant therapy and those without a nadir PSA level $<0.2 \mathrm{ng} / \mathrm{mL}$. All patients who had pathological lymph node metastasis received adjuvant therapy and they were not included in this study. In total, 1283 (73\%) patients did not receive NHT and 466 (27\%) patients did. Both open and laparoscopic RPs were performed by the retroperitoneal approach. Robot-assisted RP was basically performed by the transperitoneal approach.

BCR was defined as two successive PSA levels of $>0.2 \mathrm{ng} / \mathrm{mL}$ after RP. PSA has been measured by an ultrasensitive assay (detection limit of $0.01 \mathrm{ng} / \mathrm{mL}$ ) since 2002 and the conventional PSA assay (detection limit of $0.1 \mathrm{ng} / \mathrm{mL}$ ) was performed before that. To detect BCR, PSA was generally measured at 3-month intervals for the first 2 years after surgery, at 6-month intervals for the next 3 years, and annually thereafter, but the schedule was often modified according to several clinicopathological parameters during follow-up. During the mean follow-up period of 6.9 years, BCR developed in 240 (19\%) non-NHT patients, and in 131 (28\%) NHT patients. To reduce the heterogeneity in the type of NHT agents, we excluded 11 patients who received antiandrogen monotherapy as NHT. Finally, 240 non-NHT patients and 120 NHT patients who experienced BCR were the subjects of this study.

Prior to RP, all NHT patients received at least 3 months of hormonal therapy, including a LHRH agonist (leuprorelin acetate or goserelin acetate), or the combination of LHRH agonist and antiandrogen drug (bicalutamide, flutamide, or chlormadinone acetate), which was termed combined antiandrogen blockade (CAB). The choice of hormonal therapy and duration of NHT mainly depended on the physician and patient's preference.

The pathological diagnosis by needle biopsy and RP specimen, and clinicopathologic parameters was determined by review of the medical records. Based on the PSA increase from BCR to the initiation of salvage treatment, we calculated the PSA-DT. The PSA-DT was calculated using a formula that employs the natural logarithm of 2 divided by the slope obtained from fitting linear regression of the natural log of PSA to time [24]. Patients were divided into 6 groups by setting multiple cut-off points for the timing of BCR at clinically convenient times of $1,2,3,4$, and 5 years after surgery. We confirmed that the PSA-DTs exhibited a log-normal distribution, and the Shapiro-Wilk normality test gave a $p$ value of 0.881 for non-NHT patients (Fig. 1a) and 0.086 for NHT patients (Fig. 1b). All of the individual $p$ values were also higher than 0.05 in non-NHT patients who experienced BCR in the first year after surgery, between 1 and 2 years, between 2 and 3 years, between 3 and 4 years, between 4 and 5 years, and at $>5$ years $(0.767,0.910,0.937,0.379,0.093$, and 0.813 , respectively), and in NHT patients (0.104, $0.084,0.755,0.095,0.648$, and 0.205 , respectively). In this study, the minimum (fastest) PSA-DT was set as the onesided lower 95\% confidence limit, which we considered essential to detect an increase in PSA without delay [9].

The Chi-square test was used to examine differences in categorical variables. Differences in normally distributed continuous variables between two groups were analyzed using the Student's $t$ test. Propensity score (PS) matching analysis was performed to adjust for the difference in baseline clinical characteristics between the non-NHT cohort and the NHT cohort. The PS was estimated by a logistic regression model that included PSA at diagnosis, Grade Group of needle biopsy specimen, and clinical stage. PS matching was performed using the nearest neighbor method, with a caliper of 0.20 . In all analyses, $p<0.05$ was considered significant. Statistical analysis was performed using R software (version 3.0.2) and Microsoft Excel (version 2019). 
Fig. 1 Histogram of the PSAdoubling time distribution $\mathbf{a}$ in patients without neoadjuvant hormonal therapy (NHT) and in patients with NHT. PSAdoubling time exhibited a log-normal distribution both a in patients without NHT and b in patients with NHT. The Shapiro-Wilk normality test gave a $p$ value of 0.881 for non-NHT patients and 0.086 for NHT patients
PSA-DT (months) distribution

(a) In patients without neoadjuvant NHT

(b) in patients with neoadjuvant NHT

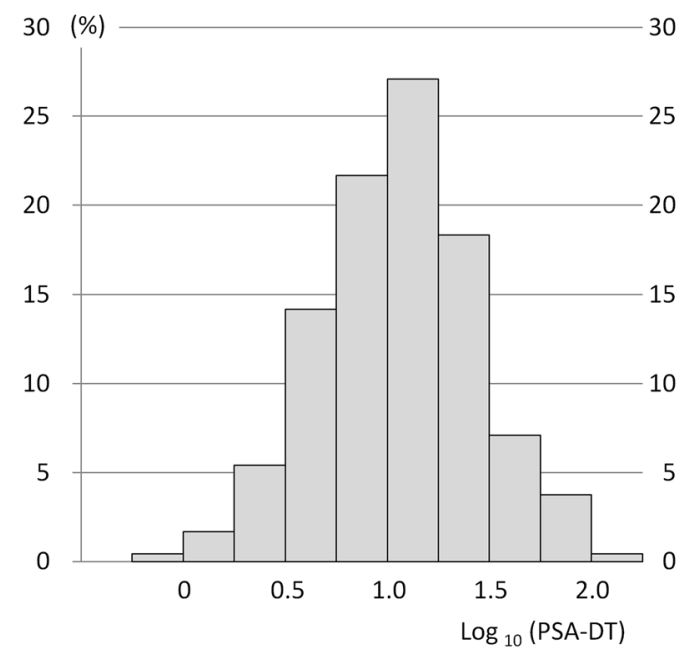

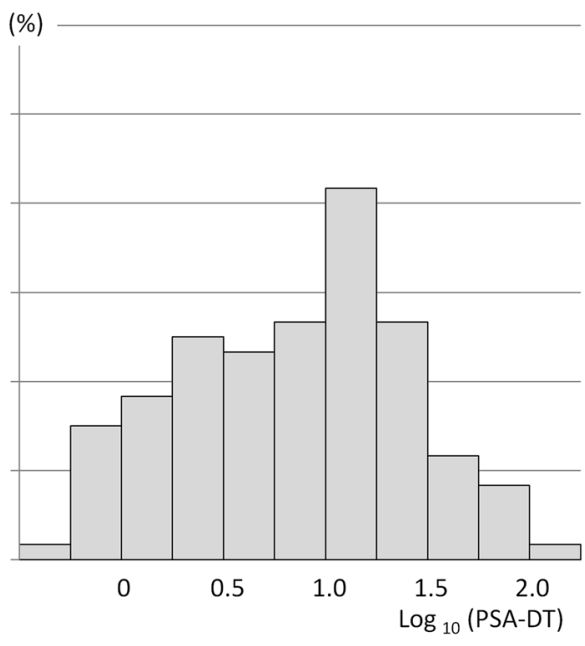

\section{Results}

\section{Patient characteristics}

The clinicopathological differences of 240 non-NHT patients and 120 NHT patients are summarized in Table 1. In the NHT cohort, the PSA level, Grade Group, and clinical stage at the diagnosis of prostate cancer were significantly higher than those in the non-NHT cohort. Regarding the type of NHT, more patients (62\%) received LHRH agonist than CAB. The duration of NHT was 3-6 months in $90 \%$ of patients and the other $10 \%$ received NHT for 7-12 months. Histological results of prostatectomy specimens revealed that organ-confined disease and negative margin status were more frequent in the NHT cohort than the non-NHT patients.

In the non-NHT cohort, $97(40.4 \%)$ and $82(34.2 \%)$ patients received salvage RT and ADT, respectively. On the other hand, $61(25.4 \%)$ received no treatment. In the NHT cohorts, $51(42.5 \%)$ and $40(33.3 \%)$ patients received salvage RT and ADT, respectively. In contrast, 29 (24.2\%) received no treatment. There was no significant difference in treatment selection against BCR between the two cohorts $(p=0.927)$. During the median follow-up period of 6.8 years after BCR, $2(0.8 \%)$ patients in the non-NHT cohort and $8(6.7 \%)$ patients in the NHT cohort died due to prostate cancer.

\section{The difference in PSA-DT following BCR}

The median PSA-DTs in non-NHT patients who experienced BCR in the first year after surgery, between 1 and 2 years, between 2 and 3 years, between 3 and 4 years, between 4 and 5 years, and at $>5$ years were $5.5,8.8,11.3,17.7,18.2$, and 18.4 months, respectively (Fig. 2a). On the other hand, those in NHT patients were 1.4, 4.1, 9.1, 13.4, 27.2, and 19.3 months, respectively (Fig. 2b). The differences in PSADTs in the first year after surgery $(p<0.001)$ and between 1 and 2 years $(p=0.005)$ were significant between non-NHT and NHT patients. On the other hand, the minimum (fastest) PSA-DTs in non-NHT patients were 1.7, 2.5, 2.9, 5.2, 7.1, and 6.2 months, respectively (Fig. 2a). Those in NHT patients were all faster than those in non-NHT patients, except for the patient who experienced BCR more than 5 years after surgery (PSA-DTs in NHT patients were 0.3 , 1.4, 1.8, 3.2, 5.8, and 7.0 months, respectively) (Fig. 2b).

After PS matching, 103 NHT patients were matched with 103 non-NHT patients, and differences in the clinicopathological background were adjusted (Supplementary Table 1). Then, a similar PSA-DT was obtained, as shown in Supplementary Fig. 1.

\section{Discussion}

The indication of hormonal therapy is generally limited to patients with metastasis, or unfavorable intermediate- or high-risk localized disease undergoing radiation therapy. In patients who receive RP, previous randomized studies demonstrated that NHT is associated with a lower positive surgical margin rate, but they failed to confirm an improved BCRfree survival or overall survival [12-14]. For these reasons, and concern about the significant side effects of hormonal therapy [11], NHT for patients who receive RP is not recommended in any guidelines [26, 27]. However, in clinical practice, there is a modest trend towards increased utilization of NHT before RP [28]. Furthermore, in this COVID-19 era, 
Table 1 Demographic and clinicopathological characteristics of 240 non-NHT patients and 109 NHT patients who experienced biochemical recurrence after surgery

\begin{tabular}{|c|c|c|c|}
\hline & $\begin{array}{l}\text { Patients without NHT } \\
N=240\end{array}$ & $\begin{array}{l}\text { Patients with NHT } \\
N=120\end{array}$ & \\
\hline PSA at diagnosis $(\mathrm{ng} / \mathrm{mL})$ & & & $p<0.001$ \\
\hline$<10$ & $134(56 \%)$ & $60(53 \%)$ & \\
\hline $10-20$ & $85(36 \%)$ & $28(25 \%)$ & \\
\hline$>20$ & $19(8 \%)$ & $26(23 \%)$ & \\
\hline Unknown & 2 & 6 & \\
\hline Grade group (needle biopsy) & & & $p=0.004$ \\
\hline 1 & $52(35 \%)$ & $19(16 \%)$ & \\
\hline 2 & $43(29 \%)$ & $34(29 \%)$ & \\
\hline 3 & $22(15 \%)$ & $24(18 \%)$ & \\
\hline 4 & $18(12 \%)$ & $19(16 \%)$ & \\
\hline 5 & $13(9 \%)$ & $22(19 \%)$ & \\
\hline Unknown & 92 & 2 & \\
\hline Clinical stage & & & $p=0.007$ \\
\hline cT1c & $38(16 \%)$ & $11(9 \%)$ & \\
\hline $\mathrm{cT} 2$ & $175(74 \%)$ & $78(67 \%)$ & \\
\hline cT3a & $23(10 \%)$ & $26(22 \%)$ & \\
\hline cT3b & 1 & $1(1 \%)$ & \\
\hline Unknown & 3 & 4 & \\
\hline Type of NHT & & & - \\
\hline LHRH agonist & - & $74(62 \%)$ & \\
\hline $\mathrm{CAB}$ & - & $46(38 \%)$ & \\
\hline Duration of NHT (months) & & & - \\
\hline 3 & - & $58(48 \%)$ & \\
\hline $4-6$ & - & $50(42 \%)$ & \\
\hline $7-12$ & - & $12(10 \%)$ & \\
\hline Grade group (prostatectomy) & & & - \\
\hline 1 & $53(22 \%)$ & - & \\
\hline 2 & $53(22 \%)$ & - & \\
\hline 3 & $82(34 \%)$ & - & \\
\hline 4 & $20(8 \%)$ & - & \\
\hline 5 & $31(13 \%)$ & - & \\
\hline Unknown & 1 & - & \\
\hline Pathological stage & & & $p<0.001$ \\
\hline pT2 & $99(41 \%)$ & $74(62 \%)$ & \\
\hline pT3a & $108(45 \%)$ & $33(28 \%)$ & \\
\hline pT3b & $33(14 \%)$ & $12(10 \%)$ & \\
\hline Undeterminable & & 1 & \\
\hline Margin status & & & $p<0.001$ \\
\hline Negative & $91(38 \%)$ & $71(60 \%)$ & \\
\hline Positive & $148(62 \%)$ & $48(40 \%)$ & \\
\hline Undeterminable & 1 & 1 & \\
\hline
\end{tabular}

NHT neoadjuvant hormonal therapy, PSA prostate-specific antigen, $L H R H$ luteinizing hormone releasing hormone, $C A B$ combined antiandrogen blockade
RP for localized prostate cancer is regarded as a non-priority surgery, and many surgeries are postponed until after the pandemic. Consequently, the number of patients who receive NHT will increase due to the fear of cancer progression during the wait times [15], although the guidelines do not recommend NHT even during the COVID-19 era [29]. Therefore, an increase in postoperative patients with NHT is of concern in the near future and the establishment of an optimal follow-up schedule for NHT patients is an urgent issue. 
Fig. 2 PSA-doubling time after biochemical recurrence at each time after surgery $\mathbf{a}$ in patients without neoadjuvant hormonal therapy (NHT) and $\mathbf{b}$ in patients with NHT. The bottom and top of the box indicate the first and third quartiles, and the band inside the box is the median. The end of the whisker represents the lower 95\% confidence limit, which is defined as the minimum (fastest) PSA-doubling time. The differences in PSA-doubling times in the first year after surgery $(p<0.001)$ and between $1-2$ years $(p=0.005)$ were significant between non-NHT and NHT patients (a) PSA-DT (months) at biochemical recurrence in patients without neoadjuvant NHT

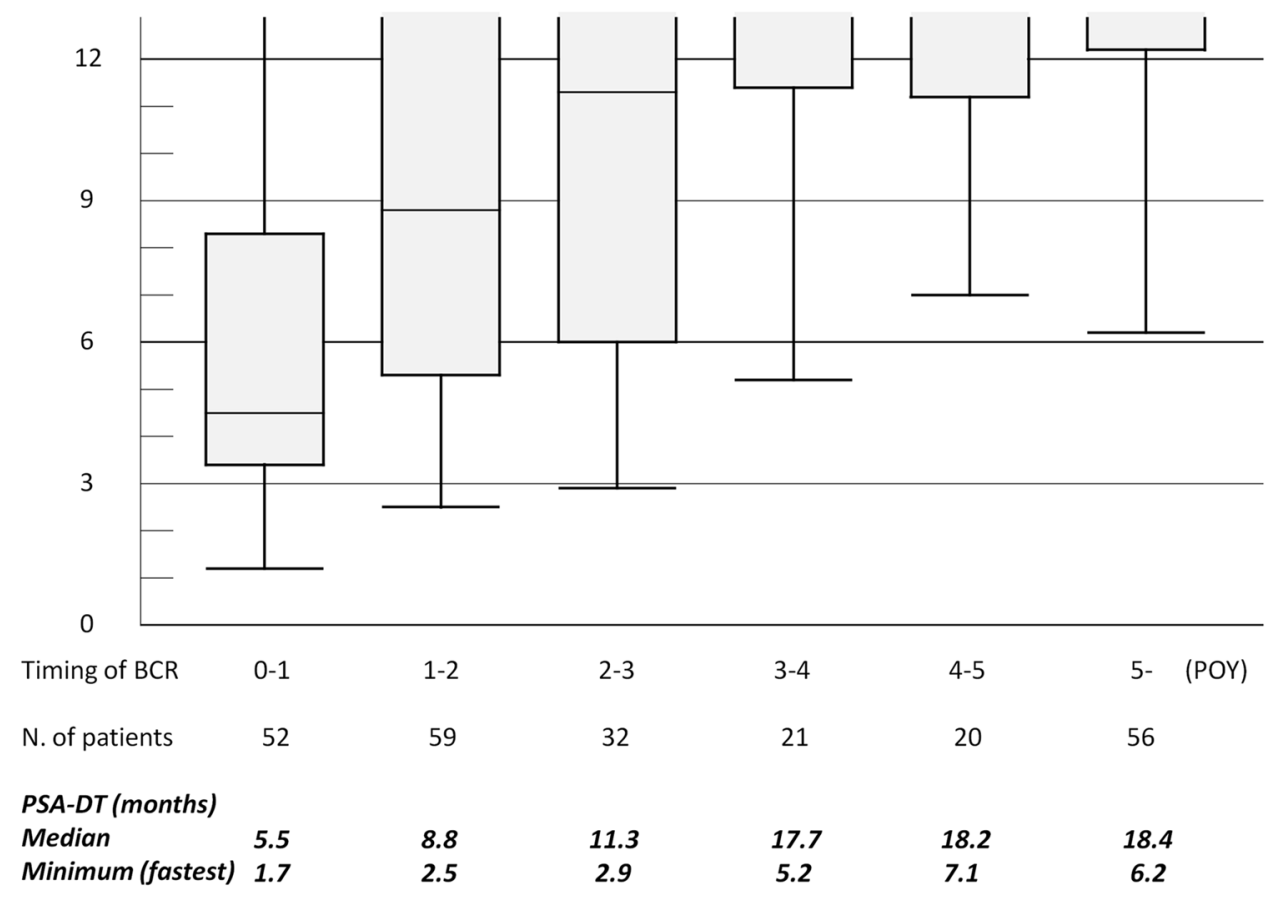

(b) PSA-DT (months) at biochemical recurrence in patients with neoadjuvant NHT

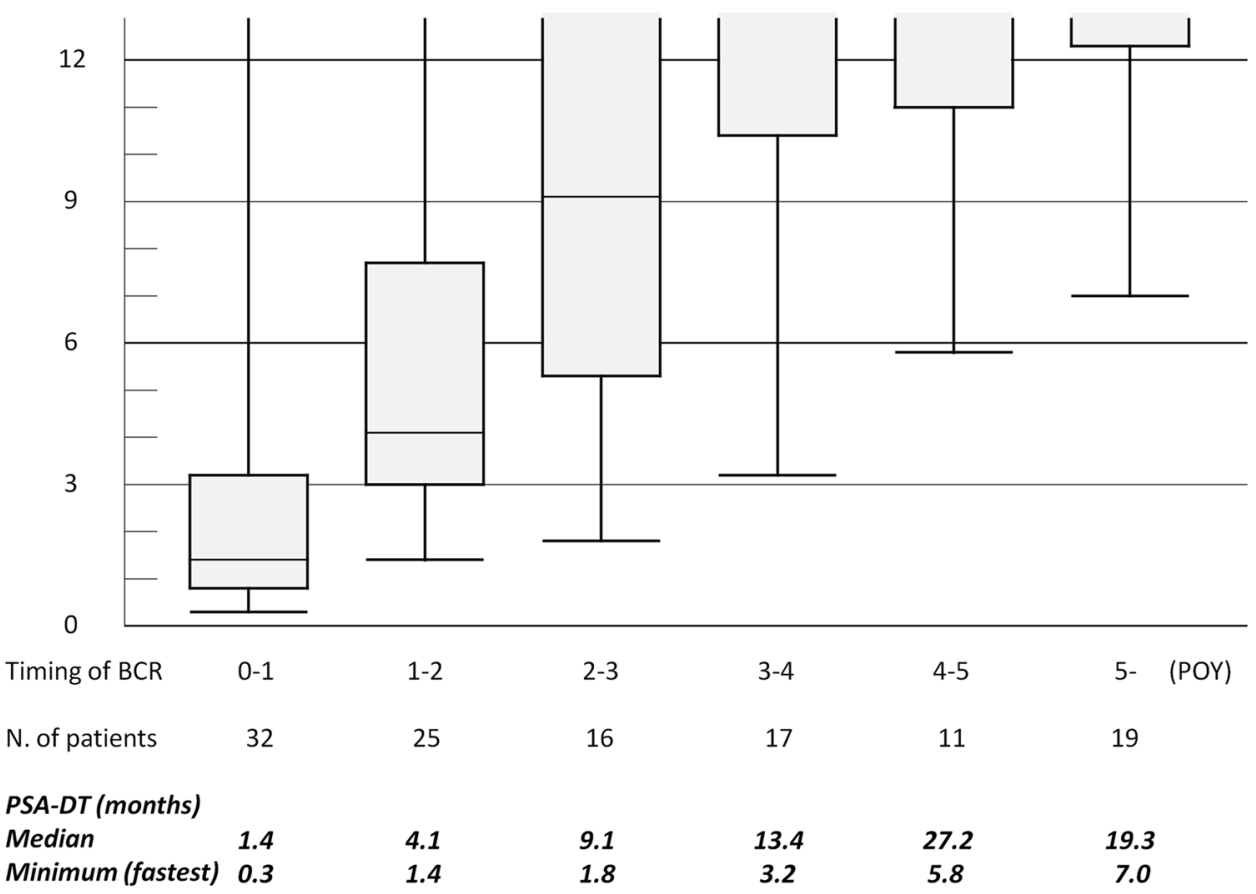

Several previous studies examined the time course and extent of testosterone recovery after the discontinuation of androgen deprivation therapy [16-23]. In half of the patients, the testosterone level recovered to a supracastrate level around 1 year after cessation of hormonal therapy, but it took an additional 1-12 months to recover to a baseline level [16-23]. Furthermore, delayed testosterone recovery is significantly associated with high age [16, 18-22], hypertension [21], diabetes [22], low baseline testosterone level [16, 18, 20-22], low serum sex hormone-binding globulin level [21], and the long duration of hormonal therapy [18-22]. However, the duration that NHT masks the increase in PSA 
Table 2 Optimal PSA follow-up schedule after radical prostatectomy

\begin{tabular}{|c|c|c|c|c|}
\hline & \multicolumn{4}{|l|}{ Timing after surgery } \\
\hline & $<1$ year & $1-2$ years & $2-3$ years & $>3$ years \\
\hline \multicolumn{5}{|c|}{ Patients without neoadjuvant hormonal therapy } \\
\hline \multicolumn{5}{|c|}{ PSA value $(\mathrm{ng} / \mathrm{mL})$} \\
\hline-0.05 & 6-month interval & 8-month interval & Annually & Annually* \\
\hline $0.06-0.10$ & 3-month interval & 4-month interval & 6-month interval & Annually \\
\hline $0.11-0.20$ & 1- or 2-month interval & 2-month interval & 3-month interval & 6-month interval \\
\hline \multicolumn{5}{|c|}{ Patients with neoadjuvant hormonal therapy } \\
\hline \multicolumn{5}{|c|}{ PSA value $(\mathrm{ng} / \mathrm{mL})$} \\
\hline-0.05 & 2-month interval & 4-month interval & Annually & Annually* \\
\hline $0.06-0.10$ & 1-month interval & 2-month interval & 6-month interval & Annually \\
\hline $0.11-0.20$ & 1-month interval & 1-month interval & 3-month interval & 6-month interval \\
\hline
\end{tabular}

The first column values are the PSA at any timing after surgery, and each box indicates the optimal interval of the next PSA measurement according to the timing after surgery

*PSA monitoring was stopped for patients who had continuously undetectable PSA levels $(<0.01 \mathrm{ng} / \mathrm{mL})$ for 5 years

due to BCR has not been examined sufficiently. In the present study, we found that NHT can conceal BCR and induce the subsequent rapid PSA increase for at least 2 years, which may prevent salvage treatment at the optimal timing.

As all patients who develop distant metastasis and die due to prostate cancer inevitably experience BCR in advance, PSA monitoring is essential for surveillance after RP. Prompt initiation of salvage therapy may prevent subsequent clinical progression and prostate cancer-specific mortality. Therefore, early detection of BCR without delay is the key in PSA follow-up after RP. We considered the ideal PSA range for the detection of BCR to be $0.2-0.4 \mathrm{ng} /$ $\mathrm{mL}$, at which we can start salvage treatment before the PSA level exceeds $0.5 \mathrm{ng} / \mathrm{mL}$ [4]. In our previous studies [8-10], we calculated the PSA-DT in patients who underwent RP between 1995 and 2017 and experienced BCR. The majority of the cohort did not undergo NHT. According to their PSADTs, we proposed the "optimal PSA follow-up schedule after RP in non-NHT patients" (the upper half of Table 2). In brief, we considered the ideal PSA range for detecting BCR to be $0.2-0.4 \mathrm{ng} / \mathrm{mL}$ to start salvage treatment before the PSA exceeds $0.5 \mathrm{ng} / \mathrm{mL}$. Therefore, the optimal PSA follow-up interval was determined to detect BCR before the PSA exceeds $0.4 \mathrm{ng} / \mathrm{mL}$. The optimal (safe) follow-up interval was calculated by estimating the timing when the PSA would reach $0.4 \mathrm{ng} / \mathrm{mL}$ based on the minimum (fastest) PSA-DT. In the present study, we found that PSA-DTs in NHT patients who experienced BCR in the first year and between 1 and 2 years after surgery were one-third and half of those in non-NHT patients, respectively. Therefore, we propose the "optimal PSA follow-up schedule after RP in NHT patients", as shown in the lower half of Table 2.

The present study has several limitations, including its retrospective design at a single institution, and limited number of patients, especially in NHT cohorts. The most important limitation of this study is that sufficient monitoring of serum testosterone was not performed in the majority, which may be a predictor for the timing of PSA increase after testosterone recovery. Simultaneous follow-up of PSA and serum testosterone is required to elucidate the impact of NHT on BCR after radical therapy. Meanwhile, it takes a few days to get the serum testosterone level after blood testing, and determining the next follow-up timing according to its result is not clinically practical. This retrospective study was conducted using PSA data for which the measurement interval in each patient was decided according to the physician's and patient's preferences, which reduces the evidence level of our study. Although our study had these limitations, our study demonstrated that patients who received NHT had a higher risk of a rapid PSA increase when they experienced BCR, especially within 2 years after RP. In order to not miss the optimal timing of salvage treatment for BCR, intensive PSA follow-up is necessary. We believe that our "optimal follow-up schedule after RP in NHT patients" may reduce the overlooking of a rapid PSA increase due to BCR. In the future, external validation with an independent data set containing a sufficient number of NHT patients is needed to confirm the safety and clinical usefulness of our follow-up schedule for PSA.

Supplementary Information The online version contains supplementary material available at https://doi.org/10.1007/s10147-021-01942-8.

Acknowledgements This research did not receive any specific grant from funding agencies in the public, commercial, or not-for-profit sectors.

Authors' contribution Conception and design: KM. Acquisition of data: KM, NN, TS. Analysis and interpretation of data: KM, TK, TT, YY, NT. Writing the manuscript: KM. Review the manuscript: KM, TK, SM, RM. Study supervision: HA, MO. 


\section{Declarations}

Conflict of interest The authors declare that they have no conflict of interest.

Ethical approval This study was approved by the institutional review board (No. 20160084).

\section{References}

1. Hamdy FC, Donovan JL, Lane JA et al (2016) 10-year outcomes after monitoring, surgery, or radiotherapy for localized prostate cancer. N Engl J Med 375(15):1415-1424

2. Kakehi Y, Sugimoto M, Taoka R, Committee for establishment of the evidenced-based clinical practice guideline for prostate cancer of the Japanese Urological Association (2017) evidenced-based clinical practice guideline for prostate cancer (summary: Japanese Urological Association, 2016 edition). Int J Urol 24(9):648-666

3. Matsumoto K, Niwa N, Hagiwara M et al (2020) Type of patients in whom biochemical recurrence after radical prostatectomy can be observed without salvage therapy. World J Urol 38(7):1749-1756

4. Pfister D, Bolla M, Briganti A et al (2014) Early salvage radiotherapy following radical prostatectomy. Eur Urol 65(6):1034-1043

5. Matsumoto K, Mizuno R, Tanaka N et al (2014) Optimal timing of hormonal therapy for prostate-specific antigen recurrence after radical prostatectomy. Med Oncol 31(7):45

6. Stish BJ, Pisansky TM, Harmsen WS et al (2016) Improved metastasis-free and survival outcomes with early salvage radiotherapy in men with detectable prostate-specific antigen after prostatectomy for prostate cancer. J Clin Oncol 34(32):3864-3871

7. van den Bergh RC, van Casteren NJ, van den Broeck T et al (2016) Role of hormonal treatment in prostate cancer patients with nonmetastatic disease recurrence after local curative treatment: a systematic review. Eur Urol 69(5):802-820

8. Matsumoto K, Komatsuda A, Yanai Y et al (2017) Determining when to stop prostate specific antigen monitoring after radical prostatectomy: the role of ultrasensitive prostate specific antigen. J Urol 197(3 Pt 1):655-661

9. Matsumoto K, Niwa N, Hattori S et al (2018) Establishment of the optimal follow-up schedule after radical prostatectomy. Urol Oncol 36(7):341.e9-341.e14

10. Yanai Y, Matsumoto K, Kosaka T et al (2020) External validation of the "optimal PSA follow-up schedule after radical prostatectomy" in a new cohort. Int J Clin Oncol 25(7):1393-1397

11. Nguyen PL, Alibhai SM, Basaria S et al (2015) Adverse effects of androgen deprivation therapy and strategies to mitigate them. Eur Urol 67(5):825-836

12. Klotz LH, Goldenberg SL, Jewett MA et al (2003) Long-term followup of a randomized trial of 0 versus 3 months of neoadjuvant androgen ablation before radical prostatectomy. J Urol 170(3):791-794

13. Gravina GL, Festuccia C, Galatioto GP et al (2007) Surgical and biologic outcomes after neoadjuvant bicalutamide treatment in prostate cancer. Urology 70(4):728-733

14. Yee DS, Lowrance WT, Eastham JA et al (2010) Long-term follow-up of 3-month neoadjuvant hormone therapy before radical prostatectomy in a randomized trial. BJU Int 105(2):185-190

15. Tandogdu Z, Collins J, Shaw G et al (2020) Management of patients who opt for radical prostatectomy during the coronavirus disease 2019 (COVID-19) pandemic: an international accelerated consensus statement. BJU Int. https://doi.org/10.1111/bju.15299 (Epub ahead of print)

16. Gulley JL, Figg WD, Steinberg SM et al (2005) A prospective analysis of the time to normalization of serum androgens following 6 months of androgen deprivation therapy in patients on a randomized phase III clinical trial using limited hormonal therapy. J Urol 173(5):1567-1571

17. Murthy V, Norman AR, Shahidi M et al (2006) Recovery of serum testosterone after neoadjuvant androgen deprivation therapy and radical radiotherapy in localized prostate cancer. BJU Int 97(3):476-479

18. Yoon FH, Gardner SL, Danjoux C et al (2008) Testosterone recovery after prolonged androgen suppression in patients with prostate cancer. J Urol 180(4):1438-1444

19. Dai B, Qu YY, Kong YY et al (2013) Kinetics of testosterone recovery in clinically localized prostate cancer patients treated with radical prostatectomy and subsequent short-term adjuvant androgen deprivation therapy. Asian J Androl 15(4):466-470

20. Tsumura $H$, Satoh $T$, Ishiyama $H$ et al (2015) Recovery of serum testosterone following neoadjuvant and adjuvant androgen deprivation therapy in men treated with prostate brachytherapy. World J Radiol 7(12):494-500

21. Nam W, Choi SY, Yoo SJ et al (2018) Factors associated with testosterone recovery after androgen deprivation therapy in patients with prostate cancer. Investig Clin Urol 59(1):18-24

22. Inoue T, Mizowaki T, Kabata D et al (2018) Recovery of serum testosterone levels and sexual function in patients treated with short-term luteinizing hormone-releasing hormone antagonist as a neoadjuvant therapy before external radiotherapy for intermediate-risk prostate cancer: preliminary prospective study. Clin Genitourin Cancer 16(2):135-141.e1

23. Nascimento B, Miranda EP, Jenkins LC et al (2019) Testosterone recovery profiles after cessation of androgen deprivation therapy for prostate cancer. J Sex Med 16(6):872-879

24. Maffezzini M, Bossi A, Collette L (2007) Implications of prostatespecific antigen doubling time as indicator of failure after surgery or radiation therapy for prostate cancer. Eur Urol 51(3):605-613

25. Patel A, Dorey F, Franklin J et al (1997) Recurrence patterns after radical retropubic prostatectomy: clinical usefulness of prostate specific antigen doubling times and log slope prostate specific antigen. J Urol 158(4):1441-1445

26. Mottet N, Cornford P, van den Bergh RCN, et al. EAU Guidelines. https://uroweb.org/guideline/prostate-cancer/. Accessed 12 Jan 2021

27. Sanda MG, Cadeddu JA, Kirkby E et al (2018) Clinically localized prostate cancer: AUA/ASTRO/SUO guideline. Part II: recommended approaches and details of specific care options. J Urol 199(4):990-997

28. McClintock TR, von Landenberg N, Cole AP et al (2019) Neoadjuvant androgen deprivation therapy prior to radical prostatectomy: recent trends in utilization and association with postoperative surgical margin status. Ann Surg Oncol 26(1):297-305

29. Rapid reaction recommendations: EAU COVID-19. https:// uroweb.org/guideline/covid-19-recommendations/. Accessed 12 Jan 2021

Publisher's Note Springer Nature remains neutral with regard to jurisdictional claims in published maps and institutional affiliations. 